Quaderni di Geografia Cahiers de Gécongaty rataphie

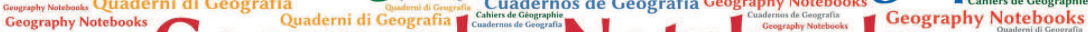
Cuadernos de Geografia Geography Notebooks $U 201000$ Cahiers de Géographi Cahiers de Géographie cahiers de Géographie Cuadernos de Geografía Cahiers de Géographie Cahiers de Géographie Cuadernos de Geografía Geography Notebooks

\author{
$4(2021)$ \\ 1 \\ Teatro di suoni. \\ Spazi acustici teatrali e territoriali
}

A cura di

Martino Mocchi, Lorena Rocca, Demis Quadri and Carlotta Sillano

EDITORIAL

Teatro di suoni per l'attaccamento ai luoghi. Uno sguardo geografico 11

Lorena Rocca

Per un teatro di suoni. Riflessioni su possibili dimensioni sonore nelle 23 creazioni site-specific di physical theatre

Demis Quadri

INTRODUCTION

Teatro di suoni. Spazi acustici teatrali e territoriali

Demis Quadri e Lorena Rocca

SPECIAL Issue

I suoni di Mantova come strumenti di interpretazione del paesaggio.

Tra turismo sostenibile ed educazione al patrimonio culturale

Valeria Pecorelli, Franca Zuccoli, Alessandra De Nicola, Enrico Squarcina

Il paesaggio sonoro campano tra contemporaneità e nuove forme

di progettualità turistica

Germana Citarella 
La narrazione spettacolarizzata del paesaggio sonoro.

Da Giuseppe Chiari a Philip K. Dick e oltre

Francesco Michi

Musica di paesaggi sonori. Enunciazione, risignificazione, comunicazione

Carlotta Sillano

Camminare per ascoltare. Partiture invisibili del territorio abitato

Elisabetta Senesi

Il paesaggio sonoro in relazione. Suono, movimento e immagini per stimolare complessità percettiva Angela Calia

Groove Fields. Understanding the Dance Floor from an Art-Based Research Perspective

Sebastian Mattbias

Il silenzio come esperienza trasformativa. L'importanza del silenzio nella meditazione e in ambito professionale

Sebastiano Caroni

Progettare il silenzio. Una lettura acustica dell'ex villaggio sanatoriale 125 Morelli a Sondalo

Martino Mocchi

Voicing One's Will. Theatre as Audio-Visual Hypotyposis of the Poetic

Michael Groneberg

Music and Clowning in Europe, 20th-21st centuries

Anna Stoll Knecht

Il paesaggio sonoro nella composizione musicale. Un percorso bibliografico

Stefano Alessandretti

\#exploreART: il labirinto di A. Pomodoro e i bambini. Un progetto di fruizione condivisa con percorsi sensoriali partecipati Alessandra De Nicola, Franca Zuccoli 


\section{OTHER EXPLORATIONS}

Il rumore lontano. Intervista a Lorena Rocca

a cura di Martino Mocchi

Re Cervo. Intervista a Antonella Astolfi

a cura di Krizia Bonaudo e Demis Quadri

Centovalli-Centoricordi. Intervista a Oliviero Giovannoni a cura di Krizia Bonaudo e Demis Quadri

Alla ricerca di un metodo: Open Space Technology 



\title{
Teatro di suoni. Spazi acustici teatrali e territoriali
}

\section{$4^{\circ}$ Workshop Internazionale sui Paesaggi Sonori}

\author{
Lorena Rocca ${ }^{1}$, Demis Quadri ${ }^{2}$ \\ ${ }^{1}$ Università degli Studi di Padova; Dipartimento DFA, SUPSI \\ ${ }^{2}$ Accademia Teatro Dimitri, SUPSI \\ DOI: https://doi.org/10.7358/gn-2021-001-roc2
}

La Società Svizzera di Studi Teatrali, in collaborazione con l'Accademia Teatro Dimitri e il Dipartimento Formazione e Apprendimento della Scuola Universitaria Professionale della Svizzera Italiana e con il sostegno della Accademia Svizzera di Scienze Umane e Sociali, nell'ambito della rassegna di manifestazioni "La Suisse existe - La Suisse n'existe pas / Raum - Espace" ha promosso la realizzazione del $4^{\circ}$ Workshop Internazionale sui Paesaggi Sonori, che si è svolto il 13 e 14 giugno 2019 tra Verscio e Locarno $(\mathrm{CH})$.

\section{TEMA DEL WORKSHOP}

La conoscenza del mondo passa attraverso il nostro corpo: la vista ha potuto sviluppare strumenti molto raffinati per immagazzinare, manipolare e interpretare i dati che la colpiscono, l'udito è "in fasce" ed è ancora alle prese con strumenti e metodi da verificare (Barbanti 2015; Bull and Back 2003; Calanchi 2015; Favaro 2010). Se non ci si può esimere dal sentire (l'apparato udivo è costantemente sollecitato anche al di là della nostra volontà) l'azione dell'ascoltare - dal latino auscultare da ausiula diminutivo di ausis (orecchio) e quindi porre l'orecchio - richiede un atto consapevole e intenzionale. La consapevolezza e l'internazionalità permettono l'avvio di processi in cui le percezioni uditive vengono poste al centro (Schäfer 1992; Schäfer 1985; Minidio 2005).

Ogni suono, infatti, reca con sé informazioni circa lo spazio nel quale 
esso prende forma, può dirci qualcosa sul luogo, i suoi abitanti, le loro attività (Pisano 2017; Calanchi and Laquidara 2017; Marchetta 2010). Il suono ci parla, ci informa, ci costringe, ci persuade a pensare e sentire (Erkizia 2017; Morelli 2010; Cox 2014). Esso racconta e ci rimanda indietro nel tempo, possiede una carica evocativa (Minidio 2005). L'esperienza e i ricordi di ogni individuo sono costellati da suoni, presenti o passati (Convery et al 2012; Truax 2008). Il suono è infatti un cronotopo, ovvero un «oggetto territoriale, che condensa un certo tempo e un certo luogo e cristallizza energia e informazione» (Rocca 2016, 82).

Esso è espressione del controllo materiale che l'uomo ha esercitato sul territorio nel tempo, ma anche riflesso dell'organizzazione di un certo periodo. Dà l'idea dell'evoluzione di un territorio. Il suono è inoltre pervasivo e occupa ogni spazio, ma, allo stesso tempo, è effimero, fuggente. Non è mai uguale, non rimane fisso, cambia continuamente; esso varia a seconda dell'ora e del posto in cui lo si ascolta (Erkizia 2017). I suoni attorno a noi permettono quindi di dare forma allo spazio che ci circonda e sono una componente fondamentale della nostra esperienza di vita (Barra and Carlo 2009, 32). Il suono contribuisce a instaurare con il luogo un legame identitario. Esso è parte costituiva della nostra cultura, tanto che viene riconosciuto dall'UNESCO come patrimonio immateriale e componente essenziale del paesaggio (UNESCO, 2003).

La Careggi Landscape Declaration on Soundscape (2012), rifacendosi alla Convenzione Europea sul Paesaggio, definisce il paesaggio sonoro come «la proprietà acustica di qualsiasi paesaggio in relazione alla percezione specifica di una specie [...], il risultato delle manifestazioni e dinamiche fisiche (geofonie), biologiche (biofonie) e umane (antropofonie)».

Molte proprietà della dimensione sonora sono anche al centro delle pratiche e delle esperienze teatrali: dalla conoscenza attraverso il corpo alla condensazione di uno spazio-tempo, dalle fondamentali interazioni con il tempo presente alla composizione antropologica di legami identitari. La dimensione sonora dell'esperienza teatrale è da sempre centrale nella cultura scenica occidentale, molto legata a una importante tradizione di prosa che ne ha definito spesso i principali sviluppi, ma non è stata adeguatamente tenuta in conto rispetto ai suoi influssi sui processi creativi e drammaturgici. Una ricerca sullo spazio dei suoni nel teatro è ancora ai suoi inizi, ma sta trovando nelle attività all'Accademia Teatro Dimitri un terreno fertile sia nelle riflessioni sul paesaggio sonoro del teatro fisico (Quadri 2017), sia in progetti come l'indagine su comicità, musica ed effetti sonori, portata avanti da Anna Stoll Knecht. 


\section{2. “COME RISUONA IL TERRITORIO NEGLI SPAZI TEATRALI?”}

Questa è stata la domanda guida del workshop, che ha catalizzato riflessioni di attori, musicisti, musicologi, geografi, storici, filosofi, pedagogisti in uno sguardo multifocale che ha preso vita a partire dalla dimensione sonora nelle pratiche ed esperienze teatrali per soffermarsi sulla modalità di conoscenza attraverso il corpo per giungere alla condensazione di uno spazio-tempo, dalle interazioni fondamentali con il tempo presente alla composizione antropologica dei legami identitari. A partire dalle riflessioni generali presenti nell'editoriale, questo volume è diviso in tre parti: 1) contributi; 2) approfondimenti; 3) questioni aperte.

Il volume si apre con uno sguardo a realtà geografiche lette e presentate attraverso le sue sonorità. In particolare i contributi di Valeria Pecorelli, Franca Zuccoli, Alessandra De Nicola, Enrico Squarcina e di Germana Citarella offrono una lettura turistica ed educativa del patrimonio sonoro di Mantova e della Campania. Il passaggio dal micro al macro è di Francesco Michi e Carlotta Sillano, che riportano l'attenzione sul paesaggio sonoro e sulla perfomatività comunicativa e drammaturgia presente in esso.

La declinazione del paesaggio sonoro nella percezione che si attua con il movimento del corpo è di Angela Calia, che indaga il tema negli ambiti della danza e del video con particolare riferimento al site-specific, e di Sebastian Matthias, che esplora il fenomeno della trasmissione del movimento nelle discoteche. La ricerca e l'esplorazione di partiture invisibile che si attuano attraverso il binomio camminare per ascoltare è quanto racchiuso nella riflessione di Elisabetta Senesi. Spostando lo sguardo dal movimento alla pausa Sebastiano Caroni e Martino Mocchi pongono al centro il silenzio come esperienza trasformativa e di progettazione architettonica.

Le forme del teatro si disegnano ulteriormente grazie ad Anna Stoll Knecht, che affronta la questione dell'umorismo musicale nel XX secolo da due diverse prospettive: da un lato, esaminando il modo in cui la musica e il suono sono usati nelle tradizioni clownistiche europee; dall'altro, proponendo di ampliare il campo dell'umorismo musicale introducendo la categoria del "clownesco". Lo sguardo si allarga quindi in unitinerario immaginario e concettuale nel quale Michael Groneberg invita a esplorare la relazione visiva e quella sonora del teatro, a partire dal mito greco.

Chiudono la sessione dei contributi la lettura del paesaggio sonoro in chiave bibliografica, proposta da Stefano Alessandretti, e il resoconto 
di un interessante progetto realizzato a Milano, a opera di Alessandra De Nicola e Franca Zuccoli.

Nella seconda parte del volume sono raccolte tre interviste a Lorena Rocca, Antonella Astolfi e Oliviero Giovannoni, che sintetizzano altrettante esperienze mosse dagli spazi e dai suoni che denotano il movimento ferroviario, un marcatore territoriale per il contesto elvetico in un gioco continuo di riferimenti al treno come spazio vivo caratterizzato da ritmi pulsanti impercettibili per chi lo vive come un luogo dove la distanza non si misura secondo una metrica spaziale, ma secondo una prospettiva temporale. La Centovallina e gli eventi scenici e artistici che l'hanno coinvolta, in particolare Il rumore lontano, Centovalli-Centoricordi e Re Cervo, sono stati anche il punto di partenza per riflessioni, basate su esperienze concrete, sulla dimensione spaziale e sonora di un modo di fare teatro fortemente radicato in un rapporto di legami antropologicamente forti con luoghi geografici e culturali di confine.

Sempre tra gli approfondimenti vengono infine sintetizzati gli esiti emersi dall'"Open Space Technology" (OST), un metodo di interazione utilizzato nelle giornate del workshop, che si basa principalmente sulla spontaneità, lasciando ai partecipanti la definizione degli argomenti da discutere. L'obiettivo principale dell'OST è quello di strutturare un ambiente di interazione che faciliti la comunicazione, costruisca una fiducia reciproca e favorisca la creatività dei partecipanti. La proposta era quella di raccogliere, in gruppi di lavoro, diverse suggestioni intorno alla domanda "Come risuona il territorio negli spazi teatrali?".

Le sintesi dei temi affrontati nei gruppi di lavoro proposti spontaneamente dai partecipanti sono intitolate: "Paesaggi sonori, linguaggi artistici e responsabilità", "Paesaggio, relazioni, genius loci e tradizioni musicali popolari", "Il lago di Salmace", "Geografia emozionale: movimento, immagine, suono" e "Il rapporto tra spazi sferici e lineari". Le discussioni all'interno dei gruppi sono state molto vivaci e arricchenti e hanno incoraggiato la possibilità di dare avvio a nuove collaborazioni e progetti.

La preservazione e valorizzazione della ricchezza, della diversità degli approcci e della cura di questo numero si deve a Martino Mocchi, che ringraziamo per il virtuoso lavoro di connessione di ambiti e contesti anche lontani. 


\section{RIFERIMENTI BIBLIOGRAFICI}

Barbanti, R. 2015. "Ecosofia sonora. Per un ascolto del paesaggio e del mondo". In A. Calanchi. ed. Il suono percepito, il suono raccontato. Teramo: Galaad, pp. 61-84.

Barra, L. and S. Carlo. 2009. "Sound and the City. Schermi, stazioni e paesaggio sonoro urbano". Comunicazioni sociali online. 1: 32-44.

Bull, M. and L. Back. eds. 2008. Paesaggi sonori. Musica, voci, rumori: l'universo dell'ascolto. Milano: il Saggiatore.

Calanchi, A. ed. 2015. Il suono percepito, il suono raccontato: Paesaggi sonori in prospettiva multidisciplinare. Teramo: Galaad.

Calanchi, A. and A. Laquidara. eds. 2017. Soundscapes and sound identities. Teramo: Galaad.

Convery, I., G. Corsane and P. Davis. 2012. Making Sense of Place: Multidisciplinary, Perspectives. Woodbridge: Boydell.

Cox, T. 2015. Pianeta acustico. Viaggio fra le meraviglie sonore del mondo. Bari: Dedalo.

Erkizia, X. 2017. Il rumore lontano. Locarno: SUPSI.

Favaro, R. 2010. Spazio sonoro: Musica e architettura tra analogie, riflessi, complicità. Venezia: Marsilio.

Marchetta, V. 2010. Passaggi di Sound Design. Riflessioni, competenze, oggetti-eventi. Milano: Franco Angeli.

Minidio, A. 2005. I suoni del mondo: Studi geografici sul paesaggio sonoro. Milano: Guerini.

Morelli, A. 2010. "Al di là del rumore e della musica”. In A. Morelli, S. Scarani, G. E. Papa. eds. "Sound design”: progettare il suono. Bologna: Pitagora. pp. 22-35.

Pisano, L. 2017. Nuove geografie del suono. Spazi e territori nell'epoca postdigitale. Milano: Meltemi.

Quadri, D. 2017. “Il paesaggio sonoro del teatro fisico". Métis: bistória \& cultura. 16 (32): 91-97. doi: 10.18226/22362762.v16.n.32.05

Rocca, L. 2016. "Storia e geografia: Giano bifronte". In C. Minelle, L. Rocca and F. Bussi eds. Storia e geografia Idee per una didattica congiunta. Roma: Carocci. pp. 79-86.

Truax, B. 2008. "Soundscape Composition as Global Music: Electroacoustic Music as Soundscape”. Organised Sound. 13(2): 103-109.

Truax, B. 2017. "Acoustic Space, Community, and Virtual Soundscapes”. In M. Cobussen, V. Meelberg and e B. Truax eds. The Routledge Companion to Sounding Art. Routledge. pp. 253-59.

UNESCO. 2003. Convenzione per la salvaguardia del patrimonio culturale immateriale. http://unescoblob.blob.core.windows.net/documenti/5934dd11-74de-483c89d5-328a69157f10/Convenzione\%20Patrimonio\%20Immateriale_ITA\%202. pdf. 
